\title{
Actividad de cletodim y haloxifop R-metil en el suelo y su efecto fitotóxico en el cultivo de maíz (Zea mays)
}

\author{
Ustarroz, D., Montoya, J., Ledda, A., Belluccini, P. A. y Cervellini, J. M.
}

\begin{abstract}
RESUMEN
Los herbicidas haloxifop R-metil y cletodim pueden persistir activos en el suelo causando efectos fitotóxicos a cultivos de gramíneas cuando son aplicados próximos a la siembra. En este trabajo se realizaron experimentos de campo en cuatro ambientes y en macetas bajo invernáculo. El objetivo fue evaluar el efecto fitotóxico de distintas dosis de haloxifop R-metil y cletodim aplicados al suelo en el cultivo de maíz y estudiar los requerimientos de riego y tiempo entre aplicación y siembra, para evitar efectos fitotóxicos. En condiciones de campo, 62,5 y 120 g.i.a. ha-1 de haloxifop R-metil y cletodim respectivamente, produjeron fitotoxicidad visual menor a $14 \%$. Con dosis superiores, en dos de los cuatro ambientes se observaron mayores niveles de fitotoxicidad (32-78 $\%)$. Ambos herbicidas redujeron la biomasa de las plantas de maíz cuando no fueron incorporados al suelo mediante riego y la siembra se realizó 14 días después de su aplicación. Cuando se realizó riego tras la aplicación de los herbicidas, siete días resultaron suficientes para evitar fitotoxicidad con 62,5 y $125 \mathrm{~g}$ i.a. ha-1 de haloxifop R-metil y $120 \mathrm{~g}$ i.a. ha ${ }^{-1}$ de cletodim. Ninguno de los tratamientos evaluados redujo la biomasa de las plantas de maíz sembradas 14 días después del riego.
\end{abstract}

Palabras clave: inhibidores de ACCasa, residualidad, fitotoxicidad, maíz.

Ustarroz, D., Montoya, J., Ledda, A., Belluccini, P. A. y Cervellini, J. M. 2017. Soil herbicidal activity of clethodim and haloxyfop R-methyl and its phytotoxic effect in maize (Zea mays) Agriscientia 34 (II): 83-89

\section{SUMMARY}

The herbicides haloxyfop R-methyl and clethodim may persist active in the soil causing phytotoxic effects to grass crops when applied close to sowing. In this work, field and greenhouse experiments were conducted to evaluate the phytotoxic potential of different rates of these herbicides in maize and to study the irrigation requirements and time between application and sowing to avoid phytotoxicity in the crop. Field experiments were carried out on four agroecological areas. Under field conditions the lower rates of both herbicides, 62.5 and $120 \mathrm{~g}^{\text {i.a. }}$ ha-1 of haloxyfop R-methyl and clethodim respectively, produced visual phytotoxicity lower than $14 \%$. At higher rates, in two of the 
four environments higher levels of phytotoxicity (32-78\%) were registered. Both herbicides reduced maize plants biomass when they were not incorporated to the soil by irrigation and the sowing was carried out 14 days after their application. When irrigation was performed after the application of herbicides, 7 days were enough to avoid phytotoxicity at 62.5 and $125 \mathrm{~g}$ i.a. ha- ${ }^{-1}$ of haloxyfop R-methyl and $120 \mathrm{~g}$ i.a. ha ${ }^{-1}$ of clethodim. None of the evaluated treatments reduced the plant biomass of maize planted 14 days after irrigation.

Keywords: Accasa inhibitors, soil residuality, phytotoxicity, maize.

D. Ustarroz: INTA, Estación Experimental Agropecuaria (EEA) Manfredi. Ruta Nacional No 9 km 636, Manfredi, Córdoba. J. Montoya: INTA, EEA Anguil "Ing. Agr. Guillermo Covas". A. Ledda: INTA, EEA Las Breñas. P. A.Belluccini: INTA, EE.A Marcos Juárez. J. M. Cervellini: INTACONICET. Correspondencia a: ustarroz.diego@inta.gob.ar

\section{INTRODUCCIÓN}

Durante los últimos doce años en Argentina se ha confirmado la evolución de biotipos resistentes a glifosato en trece especies que se comportan como malezas (REM, 2017). De estas, ocho son gramíneas y para su control se utilizan en forma frecuente graminicidas como cletodim y haloxifop R-metil. Si bien estos herbicidas son aplicados al follaje de las plantas, y su principal acción la ejercen a través de la absorción foliar, parte de los productos alcanza el suelo y pueden persistir en él causando efectos fitotóxicos a cultivos sensibles tales como el maíz (Buhler y Burnside, 1984).

Tanto el haloxifop R-metil como el cletodim inhiben la enzima acetil-CoA carboxilasa (ACCasa), la cual cataliza el primer paso de la biosíntesis de los ácidos grasos. Estos herbicidas pertenecen a las familias de los ariloxifenoxipropionatos (fops) y ciclohexanodionas (dims) respectivamente (Wenger y Niderman, 2007).

El haloxifop-metil es utilizado como éster, el cual es rápidamente transformado en el suelo a su forma ácida con actividad herbicida (Smith, 1985). El haloxifop es clasificado como no persistente en el suelo, sin embargo ocasionalmente podría tener una breve acción residual: su degradación se ha estudiado extensamente bajo condiciones de laboratorio y de campo, y aunque los estudios se han realizado principalmente con haloxifop-R-metil, son válidos para evaluar el destino ambiental de otros ésteres y del haloxifop racémico dado que la hidrólisis del éster y la inversión del estereoquímico ocurren rápidamente en el suelo (FAO/NHO, 1995). La rápida hidrólisis del éster de haloxifop-metil con vidas medias $\left(\mathrm{DT}_{50}\right)$ de pocas horas, indica que la hidrólisis es débilmente enantioselectiva; la degradación posterior de haloxifop es más lenta con $\mathrm{DT}_{50}$ de varios días. En tres suelos, S-haloxifop se convirtió rápidamente en R-haloxifop, en suelo estéril, no se observaron degradación ni inversión, lo que indica que ambos procesos están mediados biológicamente (Poiger, Müller, Buser y Buerge, 2015).

Estudios realizados con haloxifop- $\mathrm{R}$ metil éster en seis suelos de diferentes texturas, $\mathrm{pH}$ y contenidos de materia orgánica $(\mathrm{MO})$ demostraron que tiene una corta persistencia, con $\mathrm{DT}_{50}$ menores a 0.6 días $(n=7)$ (EFSA, 2009). Otros estudios estimaron la $\mathrm{DT}_{50}$ de 3,78 a 6,96 días según las dosis utilizadas de 100 a $400 \mathrm{~g}^{\text {i.a ha }}{ }^{-1}$, respectivamente (Chakraborty, Ghosh, Banerjee, Bhatiacharyya y Roy, 2005). Por otra parte, Vencil (2002), obtuvo persistencias más prolongadas con $\mathrm{DT}_{50}$ de dos a tres meses. La vida media de haloxifop en un suelo arcilloso franco, arcilloso y arenoso franco fue de 92, 38 y 28 días respectivamente, lo que pone de manifiesto que el tipo de suelo tiene una influencia significativa en las tasas de degradación. Esto podría estar relacionado con que luego de la hidrólisis y formación del ácido, este último se adsorbe fuertemente en la MO del suelo (Smith, 1985).

Existe información divergente en cuanto a la adsorción de haloxifop al suelo. Según Rao (2000), en general, los herbicidas arilfenoxi propiónicos son fuertemente adsorbidos al suelo y tienen baja solubilidad en el agua, mientras que EFSA (2009) indica que haloxifop-P es móvil a muy móvil en el suelo. Los coeficientes de adsorción de haloxifop-R metil éster no pudieron ser determinados dada la rápida mineralización, pero otros estudios de 
adsorción realizados con quince muestras de suelo o sedimento con propiedades fisicoquímicas muy variables, arrojaron un valor promedio del coeficiente de adsorción $\left(K_{d}\right)$ de $1,09 \mathrm{~mL} \mathrm{~g}^{-1}$ para haloxifop. La adsorción demostró no estar significativamente influenciada por el contenido de carbono orgánico, el contenido de arcilla o el pH (Rick, Slife y Banwart, 1987).

La literatura científica acerca del herbicida cletodim en cuanto a su comportamiento ambiental y la persistencia en el medio ambiente es bastante limitada (Sevilla-Morán, López-Goti, Alonso-Prados y Sandín-España, 2013). Es un compuesto débilmente ácido con un $\mathrm{pK}_{\mathrm{a}}$ de 4.1; clasificado como móvil a muy móvil en el suelo con valores de coeficiente de partición $\left(\mathrm{K}_{\text {oc }}\right)$ de 900 (Roberts, 1998), valores del coeficiente de Freundlich $\left(\mathrm{K}_{\mathrm{f}}\right)$ de 0.065 a $0.993 \mathrm{~mL} \mathrm{~g}^{-1}$ y de $\mathrm{K}_{\mathrm{foc}}$ de 3-43. Su adsorción demuestra una relación inversa con el pH (EFSA, 2011). En un estudio de adsorción y desorción con cinco suelos en un rango de $\mathrm{MO}$ de 0.4 a $2.8 \%$ y diferentes texturas, se hallaron valores de $K_{d}$ de adsorción de 0.08 a $1.57 \mathrm{~mL} \mathrm{~g}^{-1}$ y $\mathrm{K}_{\mathrm{d}}$ de desorción de 1.40 a $380 \mathrm{~mL} \mathrm{~g}^{-1}$ (Pack, 1988 citado por FAO/ WHO 1999).

El cletodim no es persistente en el suelo con una $\mathrm{DT}_{50}$ de alrededor de tres días (Vencil, 2002), es degradado rápidamente por hidrólisis y fotodegradación (Mónaco, Weller y Ashton, 2002; Sandín-España, Sevilla-Morán, López-Goti, Mateo-Miranda y Alonso-Prados, 2016), y existen antecedentes que indican que es transformado rápidamente al metabolito sulfóxido de clethodim tanto en suelos estériles como no estériles, detectándose la sustancia activa el día 0 en suelo no estéril, y en los días 0 y 1 en suelo estéril (FAO/ WHO, 1999). La degradación de cletodim aumenta en medios ácidos, acelerándose aún más bajo la incidencia de luz ultravioleta con una $\mathrm{DT}_{50}$ de 2.4, 2.6 y $3.2 \mathrm{~h}$ a $\mathrm{pH} 5,6$, y 7 , respectivamente. Asimismo, la adición de adyuvantes promueve la tasa de degradación (Falb, Bridges y Smith, 1990). Sevilla-Morán et al. (2013) mencionan que los productos de degradación de herbicidas pertenecientes a esta familia podrían presentar fitotoxicidad.

Algunos herbicidas que inhiben la ACCasa tienen actividad en el suelo y pueden brindar control residual de malezas. Sin embargo, para lograr un adecuado control residual, se requieren dosis dos a cuatro veces superiores a las utilizadas en aplicaciones foliares (Kells, Meggitt y Penner, 1985). Por lo tanto, la fitotoxicidad que producen estos herbicidas cuando son aplicados al suelo estará determinada por diferentes factores, como la dosis utilizada, la sensibilidad del cultivo, el tipo de suelo y las condiciones ambientales.

Son escasos los trabajos que han estudiado el riesgo asociado al uso de herbicidas inhibidores de la ACCasa previo a la siembra de maíz (Spader, Pereira Lopes, dos Santos Fabbrin, Gonçalves Mendonça y Pelissari, 2012; Mahoney, Shropshire y Sikkema, 2016). Por esta razón no se conoce con certeza el requerimiento de lluvias y días que deben transcurrir entre la aplicación de cletodim o haloxifop R-metil y la siembra del cultivo de maíz para evitar efectos fitotóxicos. El objetivo de este trabajo fue evaluar la fitotoxicidad que producen estos herbicidas aplicados en diferentes dosis sobre el suelo en el cultivo de maíz y cómo es afectada por la ocurrencia de precipitaciones y los días transcurridos entre la aplicación del tratamiento fitosanitario y la siembra del cultivo.

\section{MATERIALES Y MÉTODOS}

Ensayos de campo: potencial fitotóxico de haloxifop R-metil y cletodim en presiembra de cultivo de maíz.

Los experimentos fueron realizados en las estaciones experimentales agropecuarias de INTA Manfredi (Córdoba), Marcos Juárez (Córdoba), Las Breñas (Chaco) y Anguil (La Pampa). Las características de los suelos donde se realizaron las experiencias se muestran en la Tabla 1.

El diseño experimental fue en bloques completos al azar con tres o cuatro repeticiones, según cada localidad. Las parcelas fueron de $2 \mathrm{~m}$ de ancho por $10 \mathrm{~m}$ de largo. Inmediatamente antes de la siembra del cultivo de maíz se aplicó haloxifop Rmetil a dosis de 60, 120 y 240 g i.a. ha-1 y cletodim a 120, 240 y $480 \mathrm{~g}$ i.a. ha-1. Estas dosis representan las dosis recomendadas para gramíneas anuales, algunas gramíneas perennes como Cynodon dactylon y una dosis doble, respectivamente. Se incluyó también un tratamiento testigo sin herbicidas. Las aplicaciones de los herbicidas fueron realizadas con mochilas de presión constante provistas de pastillas abanico plano 110015, erogando un

Tabla 1: Características fisicoquímicas de los suelos estudiados (0-20 $\mathrm{cm}$ profundidad).

\begin{tabular}{|c|c|c|c|c|c|}
\hline \multirow[b]{2}{*}{ Localidad } & \multicolumn{3}{|c|}{ Textura } & \multirow[b]{2}{*}{$\mathrm{pH}$} & \multirow[b]{2}{*}{$\begin{array}{l}\mathrm{MO} \\
(\%)^{\mathrm{a}}\end{array}$} \\
\hline & $\begin{array}{c}\text { Arena } \\
(\%)\end{array}$ & $\begin{array}{r}\text { Limo } \\
(\%)\end{array}$ & $\begin{array}{c}\text { Arcilla } \\
(\%)\end{array}$ & & \\
\hline Manfredi & 32 & 49 & 19 & 6,6 & 1,6 \\
\hline Marcos Juárez & 6 & 68,9 & 25,1 & 6,4 & 3,2 \\
\hline Las Breñas & 17,2 & 47,2 & 35,6 & 7,6 & 1,3 \\
\hline Anguil & 48,2 & 40 & 11,8 & 6,6 & 2,2 \\
\hline
\end{tabular}

aMO: Materia orgánica. 
volumen de $130 \mathrm{~L} \mathrm{ha}^{-1}$ a una presión de 2 bar.

La siembra se realizó de forma directa sin previa remoción del suelo, con una distancia entre hileras del cultivo de $52 \mathrm{~cm}$. Las fechas de aplicación, siembra y emergencia, así como la densidad de siembra y el híbrido de maíz utilizado para cada localidad se presentan en la Tabla 2.

Las parcelas fueron mantenidas libres de malezas desde la siembra hasta la cosecha. Para esto, previo a la siembra del cultivo se aplicó atrazina + S-metolacloro a razón de $1350+1152 \mathrm{~g}$ i.a. ha ${ }^{-1}$ en todos los ensayos. Las precipitaciones registradas durante los experimentos fueron obtenidas de estaciones meteorológicas ubicadas dentro las estaciones experimentales de INTA de cada localidad.

A los 20 y 50 días después de la siembra (DDS) se realizaron evaluaciones de fitotoxicidad en forma visual respecto del testigo sin herbicida según la escala (0-100 \%) de ALAM (1974). En las localizaciones Manfredi y Las Breñas se determinó el rendimiento mediante la cosecha de las dos hileras centrales de cada parcela.

\section{Bioensayos}

Se realizaron dos tipos de ensayos conducidos en invernáculo en Manfredi:

A) Se aplicaron diferentes dosis de haloxifop Rmetil (60, 120 y 240 g i.a. ha-1) y cletodim $(120,240$ y $480 \mathrm{~g}$ i.a. ha ${ }^{-1}$ ) en el momento de la siembra de maíz, tras lo cual se regó. Se realizaron dos riegos por aspersión de $170 \mathrm{~cm}^{3}$ maceta $^{-1}$, equivalente a una lámina de $15 \mathrm{~mm}$ cada uno. El primer riego se realizó inmediatamente tras la aplicación de los herbicidas, mientras que el segundo se realizó tras 24 h desde la aplicación.

B) Se evaluaron dos dosis de los herbicidas: I) haloxifop R-metil (60 y 120 g i.a. ha-1) y II) cletodim (120 y 240 g i.a. ha-1); dos momentos de riego: I) riego después de la aplicación (RDA), con el mismo volumen y momento detallado en el ensayo $A$ y II) riego después de la siembra (RDS), en este caso el primer riego se hizo luego de la siembra y el segundo al día siguiente con el mismo volumen detallada en el ensayo $A$; dos fechas de siembra:
I) 7 días después de la aplicación (DDA) y II) 14 DDA.

Se utilizaron macetas de $12 \mathrm{~cm}$ de diámetro, las cuales fueron Ilenadas con suelo recolectado del mismo lote donde se realizó el ensayo de campo en Manfredi (Tabla 1). En dicho lote no se habían utilizado herbicidas residuales durante la campaña previa. El suelo se extrajo de $0-10 \mathrm{~cm}$ de profundidad y fue homogeneizado con un tamiz de malla de $1 \mathrm{~cm}$ previo a la preparación de las macetas. Una semana antes de la aplicación de los tratamientos el suelo fue regado en forma subsuperficial, poniendo las macetas en bandejas con agua para que esta ascienda por capilaridad a través de los orificios en las bases, hasta lograr un contenido de capacidad de campo. La temperatura media diaria del invernáculo fue obtenida promediando la temperatura máxima y mínima registrada con termómetro. Para incorporar al suelo los tratamientos herbicidas detallados previamente, se realizaron dos riegos por aspersión de $170 \mathrm{~cm}^{3}$ maceta $^{-1}$, equivalente a una lámina de $15 \mathrm{~mm}$ cada uno. Para esto se utilizaron botellas plásticas con pequeñas perforaciones en sus tapas. En cada maceta se sembraron cuatro semillas de maíz a $2 \mathrm{~cm}$ de profundidad. Una vez que las plántulas alcanzaron el estadio fenológico de dos hojas desplegadas, se cortaron al nivel del suelo y se midió el peso fresco de la biomasa aérea de cada maceta. La biomasa aérea ha sido un buen indicador del efecto fitotóxico de estos herbicidas en otros trabajos (Buhler y Burnside, 1984; Vianna y Fleck, 1988), debido a su traslocación por apoplasto (Faya de Falcón y Papa, 2001). El diseño experimental fue completamente aleatorizado con cuatro repeticiones y los experimentos fueron repetidos dos veces.

\section{Análisis estadístico}

Los datos fueron sometidos a análisis de la varianza mediante modelos mixtos (Di Rienzo et al., 2011). En los ensayos de campo los factores herbicida-dosis, localidad y sus interacciones fueron los efectos fijos del modelo, mientras que la interacción del bloque y localidad fue tomada como efecto aleatorio. En los ensayos en maceta

Tabla 2: Calendario de aplicación de los herbicidas, siembra y emergencia del cultivo, densidad e híbridos de maíz utilizados.

\begin{tabular}{|c|c|c|c|c|c|}
\hline Localidad & Aplicación & Siembra & Emergencia & $\begin{array}{c}\text { Densidad } \\
\left(\text { Plantas ha }^{-1}\right)\end{array}$ & Híbrido \\
\hline Manfredi & $17 / 10 / 2014$ & $17 / 10 / 2014$ & $24 / 10 / 2014$ & 67.200 & 31Y05HR(Pioneer) \\
\hline $\begin{array}{l}\text { Marcos } \\
\text { Juárez }\end{array}$ & 28/01/2015 & 28/01/2015 & 4/02/2015 & 67.200 & SPS2721TDTG (SPS) \\
\hline Las Breñas & $28 / 01 / 2014$ & 28/01/2015 & $01 / 02 / 2015$ & 67.200 & P3115H (Pioneer) \\
\hline Anguil & $15 / 01 / 2015$ & $16 / 01 / 2015$ & $24 / 01 / 2015$ & 45.000 & DK7210 (Dekalb) \\
\hline
\end{tabular}


los factores herbicida-dosis, momento de riego, fecha de siembra y sus interacciones fueron los efectos fijos del modelo, mientras que la repetición del experimento fue tomada como efecto aleatorio. La medias fueron comparadas con el test DGC (Di Rienzo, Guzmán y Casanoves, 2002).

\section{Resultados y discusión}

Ensayos de campo: potencial fitotóxico de haloxifop R-metil y cletodim en presiembra de cultivo de maíz.

Los mayores niveles de fitotoxicidad a los 20 DDA se observaron en Marcos Juárez y Anguil con las dosis medias y altas de ambos herbicidas (Tabla 3). En Anguil la fitotoxicidad se redujo significativamente a los 50 DDA, mientras que en Marcos Juárez los valores se mantuvieron altos superando el $80 \%$ de fitotoxicidad en el tratamiento de cleto$\operatorname{dim} 480 \mathrm{~g}$ i.a. ha-1. En ambas localidades se produjeron precipitaciones durante la emergencia 0 inmediatamente después de esta, siendo Marcos Juárez donde mayores precipitaciones se registraron dentro de los 15 DDA (Figura 1).

En Manfredi y Las Breñas se observaron bajos niveles de fitotoxicidad (Tabla 3). En la primera localidad esto estuvo relacionado con la fitotoxicidad que presentaron unas pocas plantas cuyas semillas germinaron después de las precipitaciones a los 15 DDA. El proceso de imbibición y germinación de estas semillas se dio en la solución edáfica con residuos de herbicidas que provocaron cierto nivel de toxicidad a dichas plántulas. Aquellas plantas de maíz que ya habían emergido en el momento de dicha precipitación no se vieron afectadas por los herbicidas. Los bajos niveles de fitotoxicidad observados en las localizaciones Manfredi y Las Breñas no afectaron al rendimiento del cultivo.

La mayor fitotoxicidad observada con las dosis más altas de ambos herbicidas coincide con

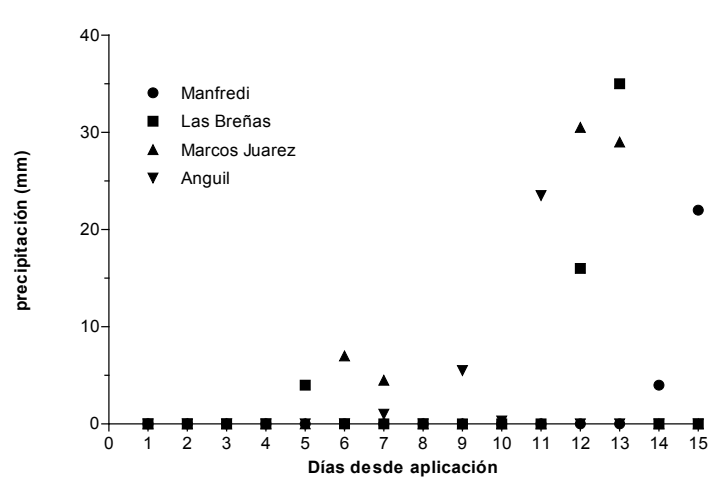

Figura 1: Precipitaciones $(\mathrm{mm})$ registradas en los días posteriores a la aplicación de los herbicidas.

los resultados de otros trabajos, en los que el incremento de la dosis de herbicidas graminicidas aplicados al suelo produjo una mayor reducción de crecimiento en distintas especies gramíneas (Buhler y Burnside, 1984; Kells et al., 1985). Los bajos valores de fitotoxicidad observados con las dosis más bajas de cletodim y haloxifop R-metil, aun en aquellos ambientes en donde las condiciones fueron propicias para la acción de los productos en el suelo (Marcos Juárez y Anguil), coinciden con los obtenidos en otros trabajos. Spader et al. (2012) encontraron que la aplicación de cletodim a 72 y $96 \mathrm{~g}$ i.a. ha ${ }^{-1}$ entre siete y dos días antes de la siembra de maíz no producía fitotoxicidad ni reducción en el rendimiento del cultivo. Resultados similares fueron obtenidos con cletodim a 30 y $60 \mathrm{~g}$

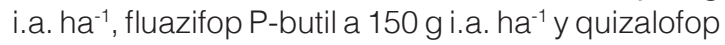
P-etil a 72 g i.a. ha-1 aplicados el día previo a la siembra de maíz, no observándose fitotoxicidad ni reducción de biomasa en el cultivo y con tan solo un $2 \%$ de pérdida en el rendimiento (Mahoney et al., 2016). Estos resultados indican que el riesgo de fitotoxicidad asociado a la aplicación de herbicidas inhibidores de la ACCasa previo a la siembra de maíz, es bajo cuando estos se utilizan

Tabla 3: Fitotoxicidad visual de maíz en diferentes localidades, evaluada a 20 y 50 días después de la siembra (DDS), en función de la dosis de cletodim y haloxifop R-metil aplicada al suelo antes de la siembra.

\begin{tabular}{|c|c|c|c|c|c|c|c|c|}
\hline \multirow[b]{3}{*}{ Tratamiento } & \multicolumn{7}{|c|}{ Fitotoxicidad (\%) } & \\
\hline & \multicolumn{2}{|c|}{ Manfredi } & \multicolumn{2}{|c|}{ Las Breñas } & \multicolumn{2}{|c|}{ Anguil } & \multicolumn{2}{|c|}{ Marcos Juarez } \\
\hline & 20DDS & 50 DDS & 20 DDS & 50 DDS & 20 DDS & 50 DDS & 20 DDS & 50 DDS \\
\hline Cletodim 480 & $2 \mathrm{c}$ & $10 \mathrm{~d}$ & $17 \mathrm{c}$ & $4 \mathrm{e}$ & $34 \mathrm{~b}$ & $7 \mathrm{e}$ & 78 a & 82 a \\
\hline Haloxifop 250 & $2 c$ & $3 \mathrm{e}$ & $2 c$ & $0 \mathrm{e}$ & $32 b$ & $14 \mathrm{~d}$ & $47 \mathrm{~b}$ & $38 c$ \\
\hline Cletodim 240 & $\mathrm{OC}$ & $0 \mathrm{e}$ & $2 c$ & $0 \mathrm{e}$ & $32 b$ & $5 \mathrm{e}$ & $57 \mathrm{~b}$ & $57 \mathrm{~b}$ \\
\hline Haloxifop 125 & $1 \mathrm{c}$ & $0 \mathrm{e}$ & $7 \mathrm{c}$ & $0 \mathrm{e}$ & $34 \mathrm{~b}$ & $10 \mathrm{~d}$ & $8 \mathrm{c}$ & $13 \mathrm{~d}$ \\
\hline Cletodim 120 & $2 c$ & $7 \mathrm{e}$ & $1 \mathrm{c}$ & $0 \mathrm{e}$ & $12 c$ & $0 \mathrm{e}$ & $5 \mathrm{c}$ & $2 \mathrm{e}$ \\
\hline Haloxifop 62,5 & $\mathrm{OCC}$ & $0 \mathrm{e}$ & $0 \mathrm{c}$ & $0 \mathrm{e}$ & $14 \mathrm{c}$ & $1 \mathrm{e}$ & $3 c$ & $2 e$ \\
\hline
\end{tabular}


a las dosis recomendadas para gramíneas anuales en estados tempranos de desarrollo.

\section{Bioensayos}

La temperatura media dentro del invernáculo entre la aplicación de los herbicidas y la siembra de maíz fue de $19,7^{\circ} \mathrm{C}$. En el ensayo $\mathrm{A}$, en el cual la siembra se realizó inmediatamente después de la aplicación de los herbicidas y su incorporación mediante riego, todos los tratamientos excepto la dosis más baja de cletodim, redujeron significativamente la biomasa aérea de las plantas de maíz (Tabla 4).

En el ensayo B, cuando el riego se realizó después la aplicación de los herbicidas, las plantas de maíz sembradas siete días después no sufrieron reducción de crecimiento con la dosis más baja de cletodim, ni con las dos dosis evaluadas de haloxifop R-metil y a los catorce DDA ninguno de los tratamientos herbicidas redujo significativamente la biomasa de las plantas. (Tabla 5). Por el contrario, cuando se regó después de la siembra, ambos herbicidas redujeron significativamente la biomasa de las plantas de maíz, siendo mayor el efecto con las dosis más altas (Tabla 5).

Tabla 4: Biomasa aérea de maíz en macetas con cletodim y haloxifop R-metil aplicados en el suelo en el momento de la siembra.

\begin{tabular}{|c|c|c|}
\hline Herbicida & $\begin{array}{c}\text { Dosis } \\
\left(\text { g i.a. ha }{ }^{-1}\right)\end{array}$ & $\begin{array}{c}\text { Biomasa fresca } \\
\left(\mathrm{g} \mathrm{maceta}^{-1}\right)\end{array}$ \\
\hline Testigo sin herbicida & - & $2,94 \mathrm{a}$ \\
\hline Cletodim & 120 & $2,75 a$ \\
\hline Cletodim & 240 & $1,36 \mathrm{~b}$ \\
\hline Haloxifop R-metil & 62,5 & $1,29 b$ \\
\hline Cletodim & 480 & $1,08 \mathrm{~b}$ \\
\hline Haloxifop R-metil & 125 & $0,88 \mathrm{~b}$ \\
\hline Haloxifop R-metil & 250 & $0,73 \mathrm{~b}$ \\
\hline
\end{tabular}

Medias seguidas de distintas letras indican que existen diferencias significativas según el test DGC $(p<0,05)$.

Tabla 5: Biomasa aérea de maíz en macetas según el momento de riego y días transcurridos entre la aplicación de los herbicidas cletodim y haloxifop R-metil y la siembra.

\begin{tabular}{|c|c|c|c|c|}
\hline \multirow[b]{3}{*}{ Tratamiento } & \multicolumn{4}{|c|}{ Biomasa fresca (g maceta-1) } \\
\hline & \multicolumn{2}{|c|}{7 DDA } & \multicolumn{2}{|c|}{14 DDA } \\
\hline & RDA & RDS & RDA & RDS \\
\hline Sin herbicida & 3,83 a & 4,66 a & 4,09 a & $4,37 \mathrm{a}$ \\
\hline Cletodim 120 & 2,79 a & 3,38 a & 3,68 a & $1,50 \mathrm{~b}$ \\
\hline Haloxifop R-metil 62,5 & 3,23 a & 3,33 a & 3,75 a & $1,88 \mathrm{~b}$ \\
\hline Cletodim 240 & $2,16 b$ & $1,86 \mathrm{~b}$ & 2,96 a & $0,73 \mathrm{c}$ \\
\hline Haloxifop R-metil 125 & 3,24 a & $0,98 \mathrm{c}$ & $2,90 \mathrm{a}$ & $1,16 \mathrm{c}$ \\
\hline
\end{tabular}

Medias seguidas de distintas letras indican que existen diferencias significativas entre tratamientos según el test DGC $(p<0,05)$. a 7DDA: Siembra 7 días después de la aplicación; b RDA: Riego después de aplicación; c RDS: Riego después de siembra.
Los experimentos en macetas muestran que tanto cletodim como haloxifop R-metil tienen potencial fitotóxico sobre las plantas de maíz cuando son aplicados en el suelo e incorporados con riego previo a la emergencia del cultivo, siendo mayor la fitotoxicidad con las dosis más altas. Estos resultados coinciden con los obtenidos en condiciones de campo y por Buhler y Burnside (1984), quienes encontraron reducciones de biomasa en plantas de maíz de 38 a 76 \% según la dosis de haloxifopmetil aplicada sobre las macetas.

La menor fitotoxicidad en condiciones de campo respecto de los ensayos en macetas con dosis bajas de los herbicidas, podría estar relacionada a una selectividad posicional ya que en el campo la siembra se realizó a $5 \mathrm{~cm}$ de profundidad, mientras que en las macetas las semillas fueron sembradas a $2 \mathrm{~cm}$ de profundidad. Estudios realizados con distintos graminicidas aplicados al suelo, demuestran que la reducción de crecimiento que produjeron los mismos en sorgo forrajero fue menor cuando las semillas del cultivo se encontraban a mayor profundidad (Buhler y Burnside, 1984).

Para evitar efectos fitotóxicos en las plantas de maíz con cletodim a 120 y haloxifop R-metil a 62,5

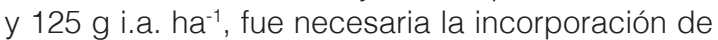
los herbicidas mediante riego y siete días a partir del mismo para realizar la siembra. Con la dosis más alta de cletodim (240 g i.a. ha-1) fueron necesarios catorce días entre el riego y la siembra para evitar efectos fitotóxicos. La incorporación de los herbicidas al suelo mediante el riego fue fundamental para la degradación de estos, ya que cuando permanecieron en la superficie del suelo por catorce días, redujeron significativamente el crecimiento de las plantas de maíz al ser incorporados en el momento de la siembra. Los resultados son de gran utilidad ya que no se dispone de información sobre los requerimientos de riego y humedad, así como del período que debe transcurrir entre la aplicación de cletodim y haloxifop R-metil y la siembra del cultivo de maíz.

\section{Agradecimientos}

Al Dr. Julio Di Rienzo por su colaboración en el análisis estadístico de este trabajo.

\section{BIBLIOGRAFÍA}

Buhler, D. D. y Burnside, O. C. (1984). Herbicidal activity of fluazifop-butyl, haloxyfop-methyl, and sethoxydim in soil. Weed Science, 32, 824-831.

Chaila, S. (1974). Resumen del panel de métodos de 
evaluación de control de malezas en Latinoamérica. Revista de la Asociación Latinoamericana de malezas, 14(2), 6-38.

Chakraborty, A., Ghosh, M., Banerjee, H., Bhatiacharyya, A. y Roy, A. (2005). Persistence behaviour of Haloxyfop - A new Herbicide in/on Onion. Journal of Crop and Weed, 1, 41-44.

Di Rienzo, J. A., Guzmán, A. W. y Casanoves, F. (2002). A multiple-comparisons method based on the distribution of the root node distance of a binary tree. Journal of Agricultural, Biological and Environmental Statistics, 7, 129-142.

Di Rienzo, J. A., Casanoves, F., Balzarini, M. G., Gonzalez, I., Tablada, M. y Robledo, C. W. (2011). InfoStat, versión 2011, Grupo InfoStat, FCA, Universidad Nacional de Córdoba, Argentina.

EFSA, (2009). Conclusion on pesticide peer review. Conclusion on the peer review of the pesticide risk assessment of the active substance haloxyfop-P (haloxyfop-R). EFSA Journal, 7, 1348.

EFSA, (2011). Conclusion on the peer review of the pesticide risk assessment of the active substance clethodim. EFSA Journal, 9, 2417.

Falb, L. N., Bridges, D. C. y Smith, Jr. A.E. (1990). Effects of $\mathrm{pH}$ and Adjuvants on Clethodim Photodegradation. Journal of Agricultural and Food Chemistry, 38, 875878.

FAO/WHO (1995). Pesticide residues in food: Evaluations. Joint Meeting of the FAO Panel of Experts on Pesticide Residues in Food and the Environment and the WHO Core Assessment Group, Geneva, 18-27 September 1995. Toxicological and environmental, part 2, 431558.

FAO/WHO (2000). Pesticide Residues in Food 1999 Evaluations: Residues, Part 1. FAO PLANT PRODUCTION AND PROTECTION PAPER 157. Clethodim (187). 117164.

Faya de Falcón, L. y Papa, J. C. (2001). El modo de acción de los herbicidas y su relación con los síntomas de daño. San Juan: INTA/EDITAR

Kells, J. J., Meggitt, W. F. y Penner, D. (1985). Activity of selective postemergence grass herbicides in soil. Weed Science, 34, 62-65.

Mahoney K. J., Shropshire, C. y Sikkema, P. H. (2016). Examining the plant-back interval for glyphosate/glufosinate-resistant corn after the application of ACCase inhibitors. Canadian Journal of Plant Science, 96, 6-10.
Mónaco, T. J., Weller, S. C. y Ashton, F. M. (2002). Weed Science: Principles and Practices. Nueva Jersey: Wiley-Blackwell.

Poiger, T., Müller, M. D., Buser, H. R. y Buerge, I. J. (2015). Environmental behavior of the chiral herbicide haloxyfop. 1. Rapid and preferential interconversion of the enantiomers in soil. Journal of Agricultural and Food Chemistry, 63, 2583-2590.

Rao, V. S. (2000). Principles of Weed Science. Oxford: Taylor \& Francis.

Rem (2017). Alertas. Recuperado el 1 de noviembre de 2017, de http://www.aapresid.org.ar/rem/alertas/

Rick, S. R., Slife, F. W. y Banwart, W. L. (1987). Adsorption of selective grass herbicides by soils and sediments. Weed Science, 35, 282-288.

Roberts, T. R. (1998). Clethodim. Metabolic Pathways of Agrochemicals. Part 1: Herbicides and Plant Growth Regulators. Londres: Royal Society of Chemistry.

Sandín-España, P., Sevilla-Morán, B. C., López-Goti, C., Mateo-Miranda, M. M. y Alonso-Prados, J. J. (2016). Rapid photodegradation of clethodim and sethoxydim herbicides in soil and plant surface model systems. Arabian Journal of Chemistry, 9, 694-703.

Sevilla-Morán, B., López-Goti, C., Alonso-Prados J. L. y Sandín-España, P. (2013). Chapter 6: Degradation of ciclohexanedione oxime herbicides. En Price, A. J. y Kelton, J. A. (Eds.). Herbicides - Advances in Research. Rijeka: InTech.

Smith, A. E. (1985). Transformation and persistence of the herbicides [14C] haloxyfop-methyl in soil under laboratory conditions. Journal of Agricultural and Food Chemistry, 33, 972-976.

Spader, V., Pereira Lopes, É. C., dos Santos Fabbrin, E. G., Gonçalves Mendonça, C. y Pelissari, A. (2012). Residual activity of ACCase inhibitor herbicides applied at pre-sowing of corn crop. Revista Brasileira de Herbicidas, 11, 42-48.

Vencill, W.K. (ed.) 2002. Herbicide Handbook. Weed Science Society of America. $8^{\text {th }}$ edition, 493pp.

Vianna, G. S. S. M y Fleck, N. G. (1988). Atividade residual de herbicidas aplicados em põs-emergéncia para controle de inços em duas condições ambientais. Pesq. Agropec. Bras, Brasilia, 23, 1095-1105.

Wenger, J. y Niderman, T. (2007). Chapter 9: Acetyl-CoA carboxylase inhibitors. En Krämer, W. y Schimer, U. (Eds.) Modern Crop Protection Compounds. Weinheim: WILEY-VCH Verlag, GmbH \& Co. 